\title{
A!
}

This is an electronic reprint of the original article.

This reprint may differ from the original in pagination and typographic detail.

Andriushchenko, E. A.; Kallaste, A.; Belahcen, A.; Heidari, H.; Vaimann, T.; Rassõlkin, A.

\section{Design optimization of permanent magnet clutch}

\section{Published in:}

Proceedings of the International Conference on Electrical Machines, ICEM 2020

DOI:

10.1109/ICEM49940.2020.9270726

Published: $23 / 08 / 2020$

Document Version

Peer reviewed version

\section{Please cite the original version:}

Andriushchenko, E. A., Kallaste, A., Belahcen, A., Heidari, H., Vaimann, T., \& Rassõlkin, A. (2020). Design optimization of permanent magnet clutch. In Proceedings of the International Conference on Electrical Machines, ICEM 2020 (pp. 436-440). [9270726] (Proceedings (International Conference on Electrical Machines)). IEEE. https://doi.org/10.1109/ICEM49940.2020.9270726

This material is protected by copyright and other intellectual property rights, and duplication or sale of all or part of any of the repository collections is not permitted, except that material may be duplicated by you for your research use or educational purposes in electronic or print form. You must obtain permission for any other use. Electronic or print copies may not be offered, whether for sale or otherwise to anyone who is not an authorised user. 
(C) 2020 IEEE. This is the author's version of an article that has been published by IEEE. Personal use of this material is permitted. Permission from IEEE must be obtained for all other uses, in any current or future media, including reprinting/republishing this material for advertising or promotional purposes, creating new collective works, for resale or redistribution to servers or lists, or reuse of any copyrighted component of this work in other works. 


\title{
Design Optimization of Permanent Magnet Clutch
}

\author{
E. A. Andriushchenko, A. Kallaste, A. Belahcen, H. Heidari, T. Vaimann, A. Rassõlkin
}

\begin{abstract}
Design optimization of permanent magnet clutches can be an effective solution for minimization of the volume at a given torque output. This study proposes an optimization method for permanent magnet clutch, which allows obtaining a complex and detailed design. Design and optimization model of the clutch, optimization method and algorithm are comprehensively discussed. Optimization using 2D finite element analysis, combined with a genetic algorithm is demonstrated. The obtained optimal shapes reduced the volume of clutch discs by $31 \%$ while the maximum integral attraction force met the limit.
\end{abstract}

Index Terms - electromagnetic coupling, design optimization, genetic algorithms.

\section{INTRODUCTION}

$\mathrm{P}$ ERMANENT MAGNET (PM) clutches have been widely applied for torque transition between two shafts without mechanical contact. The lack of mechanical contact denotes the air-gap between driving and driven member. This design feature brings several advantages to PM clutches over mechanical clutches [1], [2]. Firstly, the lack of friction reduces the risk of mechanical failures, reduces vibration and proposes easier maintenance. Additionally, the isolation of the driving and driven members is desired for a list of applications [3]-[5]. For instance, they are highly suitable for usage in a damaging, corrosive environment. Additionally, biological and food processing equipment such as mixers or agitators often requires torque to be transmitted through a separator.

One of the key requirements for clutches is a minimum volume of magnetic and non-magnetic materials with the desired torque output. These requirements can be satisfied by means of design optimization of PM clutches. So far, a considerable amount of literature has been published on design optimization of electromagnetic devices [6]-[8]. Besides, several studies have optimized design of PM clutch with different approaches [9], [10]. Up to now, most researchers have carried out optimizations using only linear dimensions as decision variables, for instance, diameters of discs or thickness of magnets [5]. However, it may be beneficial to exploit the designs that are more precise by defining optimal shapes instead of dimensions, since modern

This research work has been supported by Estonian Ministry of Education and Research (Project PSG137).

E. Andriushchenko, A. Kallaste, H. Heidari, T. Vaimann and A. Rassolkin are with the Department of Electrical Power Engineering and Mechatronics, Tallinn University of Technology, Estonia (e-mail: ekandr@taltech.ee)

A. Belahcen is with Department of Electrical Engineering and Automation, Aalto University, Espoo, Finland and with the Department of Electrical Power Engineering and Mechatronics, Tallinn University of Technology, Estonia (e-mail: anouar.belahcen@aalto.fi) manufacturing techniques allow obtaining highly complex structures. For instance, additive manufacturing provides a wide range of opportunities to more sophisticated design production. Therefore, this paper is focused on the optimization of a PM clutch shapes with the aim of obtaining the lowest weight while keeping the torque above the defined limits. As a first approximation, only shapes of the iron disks are considered in this study.

This paper begins by formulating the operation principle of the clutch and defining the design and optimization models. Later on, Section II. continues with the optimization method and algorithm description. Towards the end, results of the optimization are reported and discussed.

\section{DESIGN OPTIMIZATION}

\section{A. Design model}

The first step in any optimization is to create a design model. In this study, the design model of the clutch was made in program Finite Element Method Magnetics (FEMM). Fig. 1 illustrates the initial design of the clutch (a) and the model of the clutch (b), where magnets are highlighted in beige colour. In principle, the clutch consists of driving and driven member. The driving member possesses PM teeth placed on the disc made from printed steel. The magnets are magnetized as it is shown in Fig. 1. On the other hand, the driven member is fully made from printed steel. Working principle of PM clutch is based on attraction force between magnetic and steel teeth. The attraction force is a vector that includes two components: axial attraction force $F_{z}$ and tangential attraction force $F_{t}$. The axial attraction force allows two members to couple and move synchronously. The angle between the members and subsequent tangential attraction force are responsible for transmitted torque. This is due to the fact that the tangential attraction force arises between the members when one member spins relative to the other one. When this force reaches the maximum value, the driven member shifts to another stable position, where its teeth are aligned with the magnetic teeth of the driving member. However, if the torque on the driving member exceeds the maximum value that clutch can handle, the driven member slips. Consequently, the maximum value of the tangential attraction force defines the torque that the clutch is able to transmit [4], [11]. Therefore, the considered clutch was optimized with respect to the transmitted torque.

For a given system, the dimensions of the initial design are reported in Table I. As a first approximation, the volume of the clutch was reduced by means of the reduction of the volume of discs, particularly, by optimizing the discs 
shapes.

The flux distribution in discs in the radial direction was not changing significantly. Therefore, it was acceptable to optimize only a cross-section of a circle type (A-A) and use an obtained optimal geometry along the radial direction. In order to simplify the model for making $2 \mathrm{D}$ analysis, the cross-section was considered with straightened air gap. To simulate the geometrical continuity of each disk, periodic boundary conditions were implemented on the sides of each disk. They are shown as red and green lines in Fig. 1. The clutch was placed in the air box. The length of the disks $L$ was calculated as the length of the circle with a diameter of $D_{m}$. Consequently, the shift in this design model represented the angular deflection between clutch halves. Particularly, the used shift value was equal to $5 \mathrm{~mm}$ and denoted the position when the attraction force between the halves of the clutch was maximum. The members of the clutch were separated by the air gap equal to $1 \mathrm{~mm}$.

\section{B. Materials}

As it was mentioned before, the driving part teeth were permanent magnets, while the material of the driven member was printed steel [12]. Fig. 2 illustrates B-H magnetization curve, which characterises the magnetic properties of the utilized material. Particularly, the properties were obtained from the sample made from the gas-atomized pre-alloyed powder of Fe-Si by selective laser melting printing.

Sintered Nd-Fe-B (N52) magnets were utilized in the optimized clutch since these magnets are the strongest permanent magnets available in today's market [13]-[15].

\section{Optimization model}

The first step in optimization is to constitute an optimization model. An optimization model includes objective functions, constraints and decision variables [16] [18].

In order to obtain optimal shapes of the discs, the points on the discs' end faces were chosen as decision variables (see Fig. 3). Particularly, by changing the position of each point in $z$ and $z$ '-direction, different complex shapes could
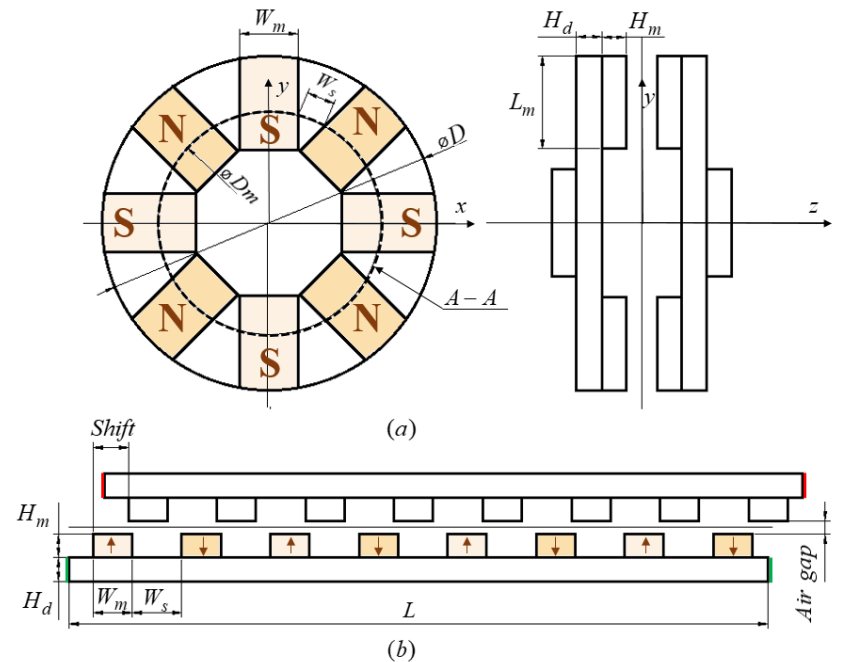

Fig. 1. Initial design (a) and model (b) of the clutch.
TABLE I

MAIN DATA OF THE CLUTCH UNDER OPTIMIZATION

\begin{tabular}{|l|c|c|}
\hline Dimension & Symbol / formula & Value \\
\hline Outer diameter & $D$ & $65 \mathrm{~mm}$ \\
\hline $\begin{array}{l}\text { Diameter in the } \\
\text { middle of magnets }\end{array}$ & $D m$ & $44.6 \mathrm{~mm}$ \\
\hline $\begin{array}{l}\text { Clutch length in } \\
\text { developed view }\end{array}$ & $L=\pi \cdot D m$ & $140 \mathrm{~mm}$ \\
\hline Magnet length & $L_{m}$ & $20 \mathrm{~mm}$ \\
\hline Magnet height & $H_{m}$ & $10 \mathrm{~mm}$ \\
\hline Magnet width & $W_{m}$ & $7.5 \mathrm{~mm}$ \\
\hline $\begin{array}{l}\text { Space between } \\
\text { magnets }\end{array}$ & $W_{s}$ & $4 \mathrm{~mm}$ \\
\hline Discs height & $H_{d}$ & 5 \\
\hline
\end{tabular}

be formed. Only six points on each disc were satisfactory $\left(p_{i}, i=1 \ldots 12\right)$, for the reason that flux lines were symmetric with respect to the axes of clutch's teeth $\left(t_{11}, t_{21}\right.$ ). The variation limits of decision variables $p_{1-6}$ in $z$ direction and $p_{7-12}$ in $z$ '-direction were $[0,3.5]$.

The objective function of the optimization model was the volume of the discs. The integral tangential attraction force $F_{t}$ was used as a constraint. During the optimization, the force was kept above $95 \%$ of the initial value with a maximum reduction of the volume. The values of objective and constraint were calculated in FEMM. The initial values of objective and constraint are reported in Table II.

\section{Optimization method and algorithm}

Optimization method can be described as a strategy or procedure within which an optimization model is optimized. Optimization method applied in this problem is called a direct optimization method since it uses design model accompanying by optimization algorithm [16], [19].

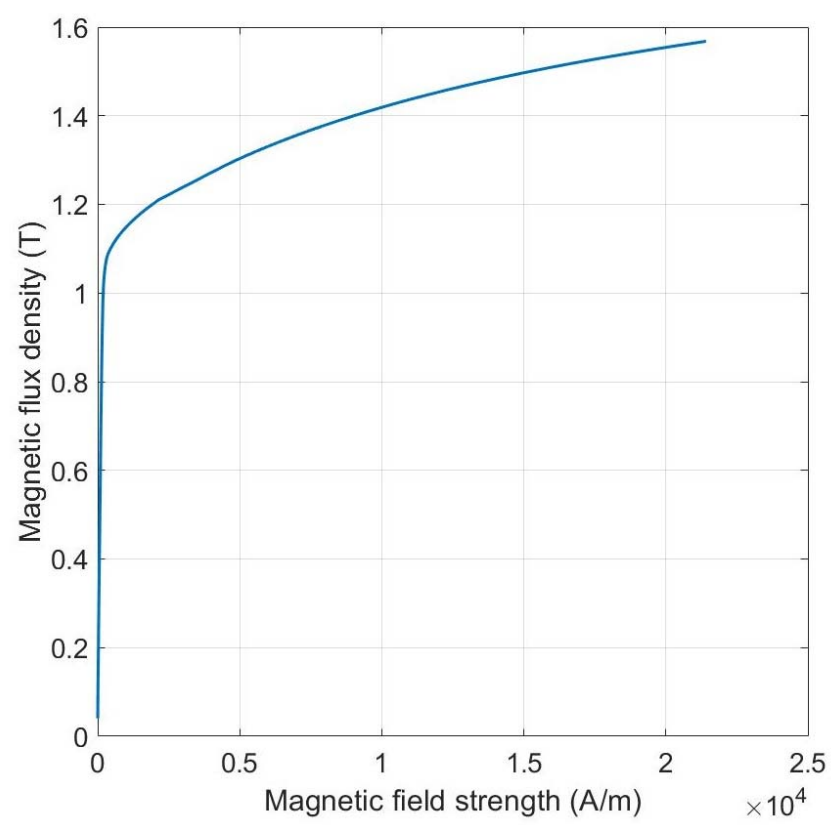

Fig. 2. B-H magnetization curve for printed steel. 


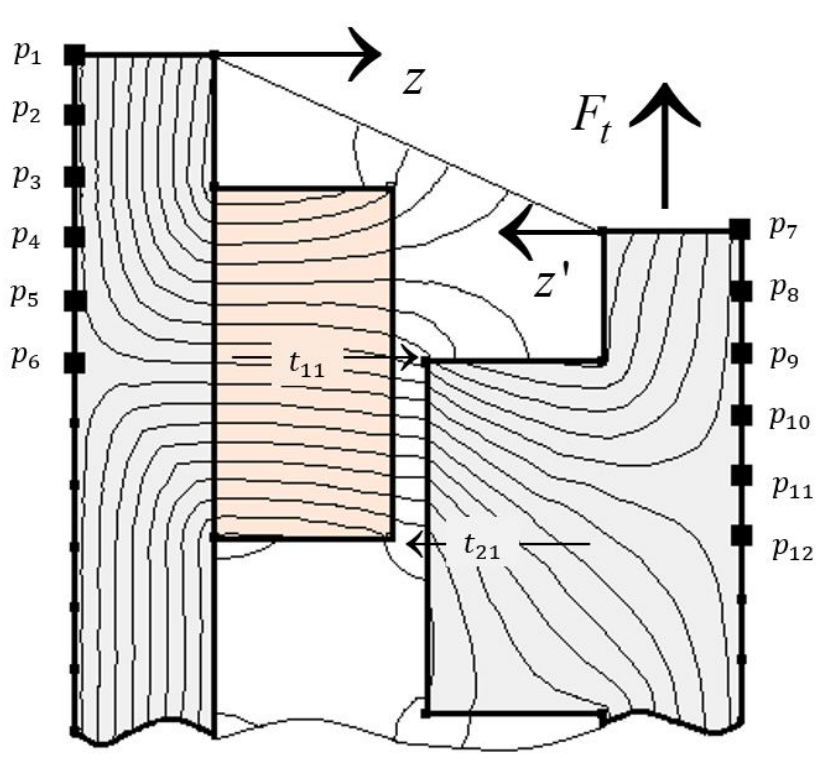

Fig. 3. Decision variables mapped on design model.

The considered optimization model had a high number of decision variables - twelve. Generally, it is rather difficult to optimize this kind of a model [16], [20]. Therefore, in order to prevent the overloading of the applied algorithm and reduce execution time, the optimization was made in two steps. More specifically, the shapes of the driving and driven disc were optimized one after another.

The optimization models were as follows:

$$
\begin{array}{ll}
\min : & \mathbf{V}_{1}\left(p_{1}, . ., p_{6}\right) \\
\text { s.t. } & \mathbf{F}_{\mathrm{t}_{1}}\left(p_{1}, . ., p_{6}\right) \geq\left[F_{t_{1}}\right],\left[F_{t_{1}}\right]=119 \mathrm{~N} ; \\
\min : & \mathbf{V}_{2}\left(p_{7}, . ., p_{12}\right) ; \\
\text { s.t. } & \mathbf{F}_{\mathrm{t}_{2}}\left(p_{7}, . ., p_{12}\right) \geq\left[F_{t_{2}}\right],\left[F_{t_{2}}\right]=117 \mathrm{~N} .
\end{array}
$$

Fig. 4 shows the applied optimization method in detail and provides the settings of the algorithm. The objective and constraint were calculated in the design model and optimization algorithm was proposing decision variables.

The algorithm used here was a single-objective genetic algorithm (GA) [21], [22]. Nowadays, GAs are widely applied due to the high number of advantages such as [23]:

1) ability to find a global minimum,

2) ability to handle non-analytic problem definition,

3) possibility to be adjusted to a particular problem,

4) robustness to noisy evaluation functions.

GA includes several operators that justify the flexibility of the algorithm as long as can be easily adjusted to a particular problem. To name, crossover, mutation, selection TABLE II

INITIAL SPECIFICATIONS OF THE CLUTCH

\begin{tabular}{|l|c|c|}
\hline Specification & Symbol/formula & Value \\
\hline $\begin{array}{l}\text { The maximum } \\
\text { integral tangential } \\
\text { attraction force }\end{array}$ & $F_{t}$ & $124 \mathrm{~N}$ \\
\hline Driving disc volume & $V_{l}$ & $11200 \mathrm{~mm}^{3}$ \\
\hline Driven disc volume & $V_{2}$ & $11200 \mathrm{~mm}^{3}$ \\
\hline
\end{tabular}

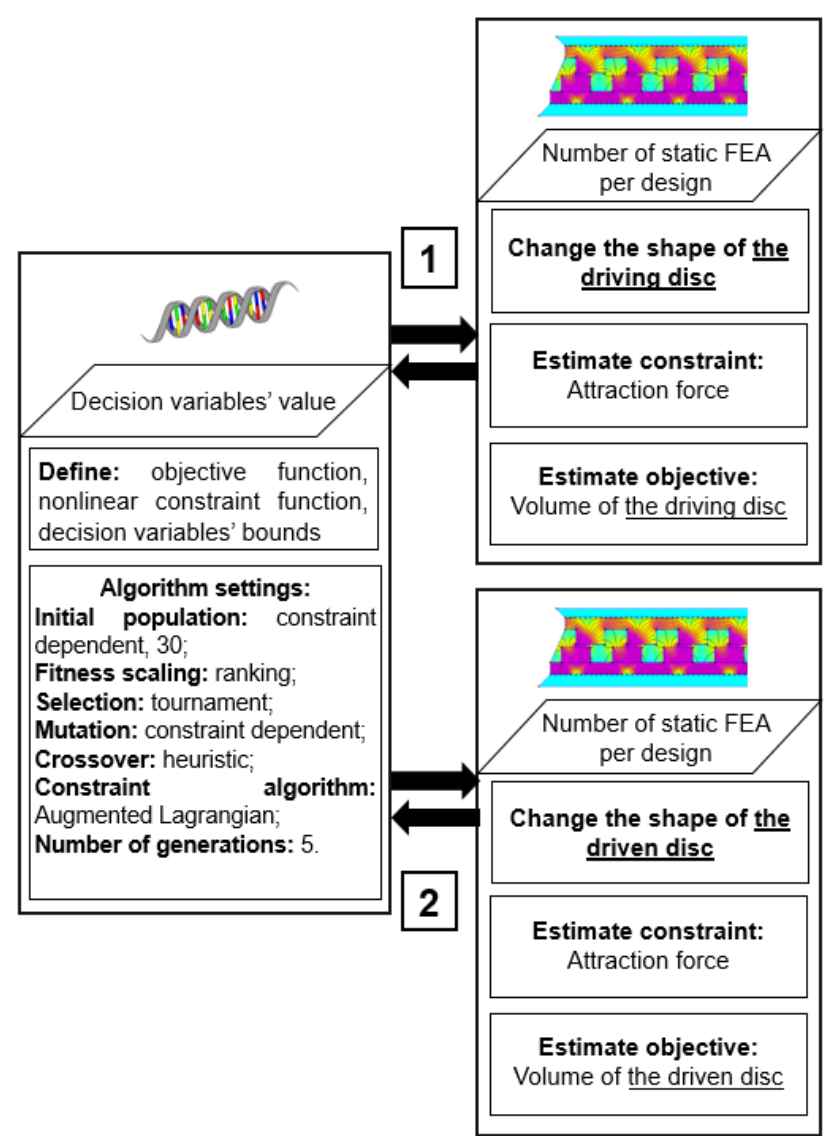

Fig. 4. Scheme of applied optimization method.

and fitness scaling are the operators. Fitness scaling is used to calculate the performance of each individual (decision variables set). The function of selection is to choose the best individuals. Further, the selected individuals constitute a new generation together with crossover fraction, which is generated by the operator crossover. This operator is applied to two individuals in order to create a new one. Finally, the operator that provides diversity and broadens the search space is mutation. It is important to mention that GA can be directly applied only to unconstrained optimization. Therefore, it is necessary to use additional techniques, such as constraint- handling methods, that can keep solutions in the feasible region [24], [25]. It is worth mentioning that algorithm settings, i.e. operators' functions, have a direct effect on optimality of the results. Therefore, for a defined problem, algorithm settings were chosen according to the common settings used in electrical devices optimization [26]-[28].

As it is shown in Fig. 4, the augmented Lagrangian was applied to handle the constraint. This method combines the objective function with constraint function using the Lagrangian [29]. Consequently, the final formulation of the problem was as follows:

$$
\mathbf{V}_{i}-\lambda_{i} \cdot s_{i} \cdot \log \left(s_{i}-\left[F_{t_{i}}\right]+\mathbf{F}_{t_{i}}\right),
$$

where the elements $\lambda_{i}>0$ are known as Lagrange multiplier estimates and the components $s_{i}>0$ are known as shifts and $s_{i}=\mu \cdot \lambda_{i}, \mu>0$. 
The number of individuals was $300(=30 \cdot 5 \cdot 2)$, where 30 is the size of each generation and 5 is the number of generations. Consequently, it required 300 evaluations of the optimization model that resulted in 4 hours of calculations.

\section{RESULTS}

Fig. 5 shows the optimal geometry of the clutch and presents the optimal values of decision variables. Overall, the volume of the driving disc was reduced significantly by $36 \%$, while the maximum value of the integral tangential attraction force met the limit $F_{t}=119 \mathrm{~N}$. The second step of optimization demonstrated considerable changes in the driven disc volume as well $(25 \%)$. Overall, there was a significant difference (31\%) between initial and optimal design volumes. What is interesting about the results is that there were no changes in the attraction force after the driven disc optimization. Therefore, it may be assumed that the geometry of the driving disc had a stronger effect on the attraction force.

In addition, the dependence of the integral attraction force on the increase in the shift was built (see Fig. 6). In Fig. 6, blue line illustrates the attraction force in initial design configuration, red line demonstrates the force after the driving disc optimization, and black line denotes the final optimized design configuration. As it was noticed before, the maximum integral attraction force did not experience radical changes after optimization. This effect remained the same way during changing of the shift.

\section{CONCLUSION}

The purpose of the current study was to optimize PM clutch shapes in order to obtain the lowest weight while keeping the torque above the defined limits.

The problem had a single-objective optimization model, where the volume of clutch's discs was the objective and the tangential attraction force was considered as a constraint. The applied method was based on the interaction between the design model and the genetic algorithm. The optimization was made in two steps, i.e. the driving and driven disc were optimized separately. It allowed preventing the overloading of the applied algorithm and reducing execution time.

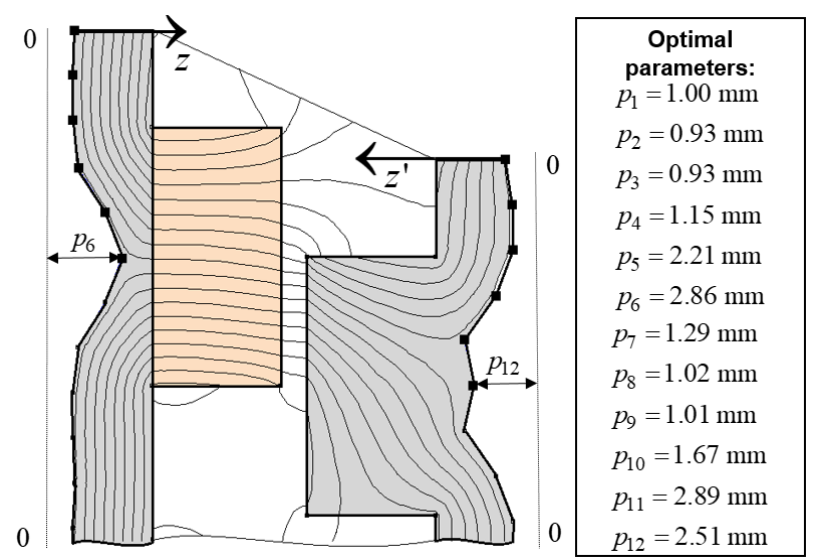

Fig. 5. Optimized geometry and optimal values of parameters.

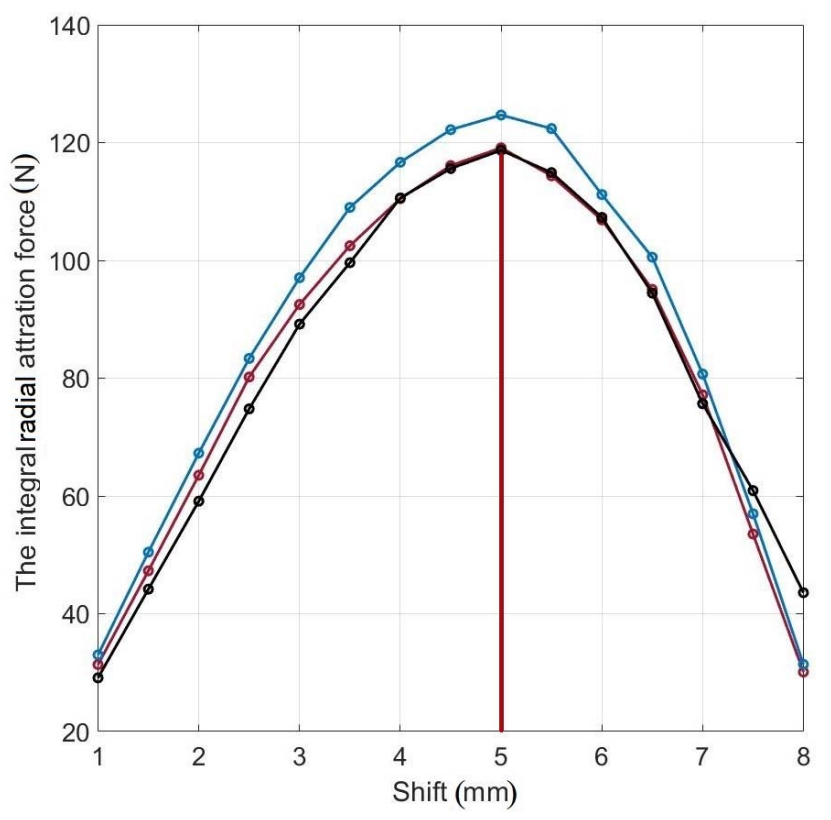

Fig. 6. Optimization results. Blue: the initial design. Red: the driving disc optimization. Black: the final optimized design.

The result of this optimization was the significant reduction in the volume of the discs $(31 \%)$, while the tangential attraction force experienced a small change from the initial value.

The present study lays the groundwork for future research into PM clutches optimization. Specifically, a natural progression of this work is to obtain optimal shapes of the permanent magnets, teeth, and discs in 3D.

\section{REFERENCES}

[1] R. Ravaud, V. Lemarquand, and G. Lemarquand, "Analytical design of permanent magnet radial couplings," IEEE Trans. Magn., vol. 46, no. 11, pp. 3860-3865, 2010.

[2] C. Ferreira, J. Vaidya, and S. Corporation, "Torque Analysis of Permanent Magnet Coupling Using 2D and 3D Finite Elements Methods," vol. 25, no. 4, pp. 3080-3082, 1989.

[3] D. Weinmann and H. J. Wiesmann, "Application of rare earth magnets to coaxial synchronous couplings," J. Magn. Magn. Mater., vol. 9, no. 1-3, pp. 179-181, Oct. 1978.

[4] K. J. Overshott, "The comparison of the pull-out torque of permanent magnet couplings predicted theoretically with experimental measurements," IEEE Trans. Magn., vol. 25, no. 5, pp. 3913-3915, 1989.

[5] W. Wu, H. C. Lovatt, and J. B. Dunlop, "Analysis and design optimisation of magnetic couplings using $3 \mathrm{D}$ finite element modelling," IEEE Trans. Magn., vol. 33, no. 5 PART 2, pp. 4083 4085, 1997

[6] C. López-Torres, A. G. Espinosa, J. R. Riba, and L. Romeral, "Design and optimization for vehicle driving cycle of rare-earth-free SynRM based on coupled lumped thermal and magnetic networks," IEEE Trans. Veh. Technol., 2018.

[7] G. Lo Calzo and M. Galea, "Design Optimization of a High-Speed Synchronous," IEEE Trans. Ind. Appl., vol. 54, no. 1, pp. 233-243, 2018.

[8] C. Babetto, G. Bacco, and N. Bianchi, "Synchronous Reluctance Machine Optimization for High-Speed Applications," IEEE Trans. Energy Convers., vol. 33, no. 3, pp. 1266-1273, Sep. 2018.

[9] R. M. Hornreich and S. Shtrikman, "Optimal design of synchronous torque couplers," IEEE Trans. Magn., vol. 14, no. 5, pp. 800-802, 1978.

[10] P. Eliès and G. Lemarquand, "Analytical optimization of the torque of a permanent-magnet coaxial synchronous coupling," IEEE Trans. Magn., vol. 34, no. 4 PART 2, pp. 2267-2273, 1998. 
[11] W. Tong, Mechanical design of electric motors. 2014.

[12] H. Tiismus, A. Kallaste, T. Vaimann, A. Rassolkin, and A. Belahcen, "Axial Synchronous Magnetic Coupling Modeling and Printing with Selective Laser Melting," 2020, pp. 1-4.

[13] H. Nakamura, K. Hirota, M. Shimao, T. Minowa, and M. Honshima, "Magnetic properties of extremely small Nd-Fe-B sintered magnets," in IEEE Transactions on Magnetics, 2005, vol. 41, no. 10, pp. 3844 3846 .

[14] Y. Matsuura, "Recent development of Nd-Fe-B sintered magnets and their applications," J. Magn. Magn. Mater., vol. 303, no. 2 SPEC. ISS., pp. 344-347, Aug. 2006.

[15] K. Hono and H. Sepehri-Amin, "Strategy for high-coercivity Nd-FeB magnets," Scr. Mater., vol. 67, no. 6, pp. 530-535, Sep. 2012.

[16] G. Lei, J. Zhu, Y. Guo, C. Liu, and B. Ma, "A review of design optimization methods for electrical machines," Energies, vol. 10, no. 12, 2017.

[17] R. C. Eberhart and Y. Shi, "Particle swarm optimization: Developments, applications and resources," in Proceedings of the IEEE Conference on Evolutionary Computation, ICEC, 2001, vol. 1, pp. 81-86.

[18] P. Ponomarev, I. Petrov, N. Bianchi, and J. Pyrhonen, "Selection of Geometric Design Variables for Fine Numerical Optimizations of Electrical Machines," IEEE Trans. Magn., vol. 51, no. 12, Dec. 2015.

[19] D. S. Weile and E. Michielssen, "Genetic algorithm optimization applied to electromagnetics: A review," IEEE Trans. Antennas Propag., vol. 45, no. 3, pp. 343-353, 1997.

[20] G. Lei, J. Zhu, and Y. Guo, Multidisciplinary Design Optimization Methods for Electrical Machines and Drive Systems. 2016.

[21] K. Deb, A. Pratap, S. Agarwal, and T. Meyarivan, "A fast and elitist multiobjective genetic algorithm: NSGA-II," IEEE Trans. Evol. Comput., vol. 6, no. 2, pp. 182-197, Apr. 2002.

[22] G. B. L. Carlos A. Coello Coello, David A. Van Veldhuizen, Evolutionary Algorithms for Solving Multi-Objective Problems. Springer US, 2007.

[23] B. Xin, L. Chen, J. Chen, H. Ishibuchi, K. Hirota, and B. Liu, "Interactive multiobjective optimization: A review of the state-of-theart," IEEE Access, vol. 6. Institute of Electrical and Electronics Engineers Inc., pp. 41256-41279, 17-Jul-2018.

[24] A. Golchha and S. G. Qureshi, "Non-Dominated Sorting Genetic Algorithm-II-A Succinct Survey," Int. J. Comput. Sci. Inf. Technol., vol. 6, no. 1, pp. 252-255, 2015.

[25] A. Jayswal and Preeti, "An exact 11 penalty function method for multi-dimensional first-order PDE constrained control optimization problem," Eur. J. Control, 2019.

[26] G. Lei, K. R. Shao, Y. Guo, J. Zhu, and J. D. Lavers, "Improved sequential optimization method for high dimensional electromagnetic device optimization," in IEEE Transactions on Magnetics, 2009, vol. 45, no. 10, pp. 3993-3996.

[27] G. Lei, K. R. Shao, Y. Guo, J. Zhu, and J. D. Lavers, "Sequential optimization method for the design of electromagnetic device," in IEEE Transactions on Magnetics, 2008, vol. 44, no. 11 PART 2, pp. 3217-3220.

[28] K. T. Alfriend, S. R. Vadali, P. Gurfil, J. P. How, and L. S. Breger, "The Basics of Analytical Mechanics, Optimization, Control and Estimation," in Spacecraft Formation Flying, Elsevier, 2010, pp. 39-57.

[29] A. R. Conn, N. Gould, and P. L. Toint, "A globally convergent Lagrangian barrier algorithm for optimization with general inequality constraints and simple bounds," Math. Comput., vol. 66, no. 217, pp. 261-289, Jan. 1997.

\section{BIOGRAPHIES}

Ekaterina Andriushchenko received her BSc and MSc degrees in mechanical engineering from Peter the Great St. Petersburg Polytechnic University, Russia, in 2013 and 2019 respectively. She is currently working toward the Ph.D. degree in electrical engineering with Tallinn University of Technology.

Her current research interest is design optimization of electrical machines.

Ants Kallaste received his $\mathrm{BSc}, \mathrm{MSc}$ and $\mathrm{PhD}$ degrees in electrical engineering from Tallinn University of Technology, Estonia, in 2004, 2006 and 2013 respectively. He is currently a senior researcher in Tallinn
University of Technology, Department of Electrical Power Engineering and Mechatronics. He is holding the position of Head of Electrical Machines Research Group. He is the member of IEEE and Estonian Society of Moritz Hermann Jacobi.

His main research interest is design of electrical machines.

Anouar Belahcen was born in Essaouira. Morocco, in 1963. He received the BSc degree in physics from the University Sidi Mohamed Ben Abdellah, Fes, Morocco, in 1988 and the MSc (Tech.) and Doctor (Tech.) degrees from Helsinki University of Technology, Finland, in 1998, and 2004, respectively. He is the professor of electrical machines at Tallinn University of Technology, Estonia and in 2013 he became Professor of Energy and Power at Aalto University, Finland.

His research interest are modeling of electrical machines, magnetic materials, coupled magnetic and mechanical problems and magnetostriction.

Hamidreza Heidari was born in Zanjan, Iran, in 1989. He received the B.Sc. and M.Sc. degrees in electronic engineering from the University of Zanjan, Zanjan, Iran. He is currently working toward the Ph.D. degree in electrical engineering with Tallinn University of Technology.

His current research interests include analysis and control of electric machines.

Toomas Vaimann received his $\mathrm{BSc}, \mathrm{MSc}$ and $\mathrm{PhD}$ degrees in electrical engineering from Tallinn University of Technology, Estonia, in 2007, 2009 and 2014 respectively. He is currently a senior researcher in Tallinn University of Technology, Department of Electrical Power Engineering and Mechatronics. He has been working in several companies as an electrical engineer. He is the member of IEEE, Estonian Society of Moritz Hermann Jacobi and Estonian Society for Electrical Power Engineering.

His main research interest is the diagnostics of electrical machines.

Anton Rassõlkin received the $\mathrm{Ph}$. D. degree in electric drives and power electronics from Tallinn University of Technology in 2014. He works as a Research Scientist at the Department of Electrical Power Engineering and Mechatronics at Tallinn University of Technology.

His main research interests are in the field of electric drives and their control systems as well as in the fields of electrical machines and electric transportation. 